\title{
Micro-colony observation: A rapid and simple approach to count bacterial colony forming units
}

Shuvam Bhuyan a, Mohit Yadav ${ }^{\mathrm{a}}$, Shubhra Jyoti Giri ${ }^{\mathrm{a}}$, Sharmilee Sarkara, Shuhada Begum ${ }^{\mathrm{a}}$, Anurag Jayswal ${ }^{\mathrm{a}}$, Kristi

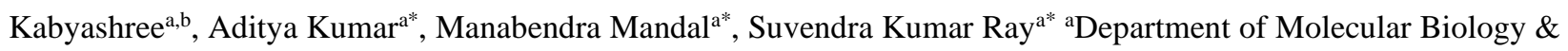
Biotechnology, Tezpur University, Tezpur-784028, Assam, India.

${ }^{b}$ National Institute of Plant Genome Research, Aruna Asaf Ali Marg, New Delhi-110067, Delhi, India. (Present

address)

"Corresponding authors: Aditya Kumar: aditya@tezu.ernet.in;

Manabendra Mandal: mandal@tezu.ernet.in;

Suvendra Kumar Ray: suven@tezu.ernet.in

\begin{abstract}
For enumerating viable bacteria, traditional dilution plating to count colony forming units (CFU) has always been the preferred method in microbiology owing to its simplicity, albeit laborious and time-consuming. Similar CFU counts can be obtained by quantifying growing microcolonies in conjunction with the perks of a microscope. Here, we employed a simple method of five microliter spotting of differently diluted bacterial culture multiples times on a single petri plate followed by finding out CFU by counting microcolonies using phase contrast microscope. In this method within four-hour period CFU of an Escherichia coli culture can be found out. Further, within ten-hour period, CFU in a culture of Ralstonia solanacearum, a bacterium with generation time around $3 \mathrm{~h}$, can be estimated. The CFU number determined by microcolonies observed is comparable with that obtained by the dilution plating method. Microcolonies number observed in the early hours of growth $(2 \mathrm{~h}$ in case of $E$. coli and $8 \mathrm{~h}$ in case of $R$. solanacearum) were found to remain consistent at later $\mathrm{h}$, though there was noticeable increase in size of the colonies. It suggested that microcolonies observed in the early hours indeed represent the bacterial number in the culture. Though manual, the method is less labor intensive apart from being simple, easy, economical and rapid.
\end{abstract}

Keywords: Colony forming unit; Microcolony; Microspoting; Escherichia coli; Ralstonia solanacearum; Phase contrast microscopy.

\section{Introduction}

Quantifying bacterial number by colony forming units (CFU) by employing serial dilution and spread plate techniques is an age-old fundamental microbiology technique used in research and diagnostics [1-3]. Though popular among the microbiologists because of its ease, the method demands large volumes of growth media, time and labor. Besides, working with bacteria having high doubling time such as Ralstonia solanacearum that takes $\sim 48 \mathrm{~h}$ to form a full-grown colony on solid agar nutrient medium only doubles the misery [4,5]. Several other methods for enumerating the number of bacteria in a culture have come to light over the years. These include the measurement of absorbance using spectrophotometer or plate reader; flow cytometry, microscopy in conjunction with staining, qPCR and several microarray-based methods with each method having its own advantages over others [6-9]. Despite all advancements 
and fast approaches to enumerate bacteria in a culture $[1,2,6]$, CFU counting has been the most dependable and popular method among microbiologists because of its viable nature and visual impact.

Therefore, it will be a big boon for the microbiologists to count CFU in a bacterial culture with reduced labor, time and cost.

In this study we are describing a subtle method of enumerating bacteria in a culture by observing microcolonies under the microscope. The basic principle of this method is to spot a small amount $(\sim 5.0 \mu 1)$ of a serially diluted bacterial culture followed by imaging and quantification of the micro-colonies obtained using the microscope [10]. In an E. coli culture, CFU can be found out within four hours after observing the microcolonies by this method. Similarly, in case of $R$. solanacearum, a bacterium with generation time more than $3.0 \mathrm{~h}, \mathrm{CFU}$ can be found out within ten hours after observing the microcolonies. The method is simple to use, scalable and costeffective that maintains the benefits of a microscopic platform. Further, validation of this method was done by comparison with other standard quantification approaches. It is pertinent to note that the microcolonies count method is done by spotting a few microliters, which enables us to analyse different dilutions in a single petridish. Therefore, the number of CFU observed under the microscope can be confirmed by observing the CFU through naked eyes in later days depending upon the growth rate of the bacteria.

\section{Materials and methods}

\section{Strains and growth conditions}

Escherichia coli DH5 $\alpha$ cells were grown in Luria-Bertani (LB) medium at $37^{\circ}$ C. R. solanacearum F1C1 (1) cells were grown in BG medium (contains $1 \%$ peptone, $0.1 \%$ yeast extract and $0.1 \%$ casamino acids) supplemented with $5 \mathrm{~g} / \mathrm{L}$ glucose at $28^{\circ} \mathrm{C}$. For solid medium agar $1.5 \%$ was used.

\section{Colony-forming unit (CFU) count}

The bacterial strains were inoculated in their respective liquid medium and incubated at their optimal growth conditions for $6 \mathrm{~h}$ in $E$. coli and overnight in $R$. solanacearum with $150 \mathrm{rpm}$ shaking condition. For counting CFU, the overnight grown culture of $E$. coli cells was serially diluted and spotted on LB agar plates as illustrated in Fig. 1. First, $100 \mu \mathrm{L}$ overnight grown culture of $E$. coli was diluted in $900 \mu \mathrm{L}$ of sterile distilled water. Since the dilution was one part sample to nine parts diluents, it was referred to as a $10^{1}$ dilution. Further, we took $100 \mu \mathrm{L}$ of the $10^{1}$ dilutions and added it to $900 \mu \mathrm{L}$ of the sterile distilled water. This dilution was a $10^{2}$ dilution. Lastly, we made a $10^{10}$ dilution by taking $100 \mu \mathrm{L}$ of the $10^{9}$ dilutions and adding $900 \mu \mathrm{L}$ of the sterile distilled water. For each dilution, we now had 1 $\mathrm{mL}$ of diluted bacterial culture. After two hours of incubation at $37^{\circ} \mathrm{C}$, micro-colonies were counted by using Life technologies EVOS FL inverted microscope with 40X magnification using the 4/10 PH condenser annulus. Under the microscope, the area covered by the cells was calculated by extrapolating the length of the $1000 \mu \mathrm{m}$ scale provided. Thus, we calculated the total CFU in the area covered by $5 \mu \mathrm{L}$ spot and subsequently in $1 \mathrm{~mL}$ of $E$. coli culture. 
This micro-colony counting was further validated by on-plate CFU counting of overnight incubation at $37^{\circ}$ C. The mean value of ten independent spots was used to show the growth parameters. Similar approach was used to count CFU in case of $R$. solanacearum culture. However, the culture was incubated at $28^{\circ} \mathrm{C}$. As $R$. solanacearum is a slow growing bacterium with a doubling time of more than $\sim 3.0 \mathrm{~h}$, its microcolonies at different dilution could be observed distinctly at $40 \mathrm{X}$ magnification in $10.0 \mathrm{~h}$ of incubation after spotting. The bacterial CFU was also counted on Life technologies EVOS FL inverted microscope with 40X magnification using the 4/10 PH condenser annulus.

\section{CFU from microcolonies method}

Microcolonies observed under the microscope of different dilutions such as $10^{4}, 10^{5}, 10^{6}$-fold etc. were counted at different time intervals manually. In case of E. coli observation of microcolonies was done from $2.0 \mathrm{~h}$ onwards up to $8.0 \mathrm{~h}$ at every $2.0 \mathrm{~h}$ intervals in the EVOS FL microscope with an objective $4 \mathrm{X}$ magnification. The area covered under the microscope was calculated from the scale bar $1000 \mu \mathrm{m}$ provided in the microscope. Total area covered under the microscope was calculated as $5.67 \mathrm{~mm}^{2}$. A $5 \mu \mathrm{l}$ culture covers an average area of $19.62 \mathrm{~mm}{ }^{2}(\mathrm{a}$ circle of average diameter of $5.0 \mathrm{~mm}$ ). From a single spot of bacterial culture, a minimum of three sections (each 5.67 $\mathrm{mm}^{2}$ ) were counted and the average number of colonies per section was found out, which was then multiplied with 3.46 to find out number of bacterial colonies in $5.0 \mu \mathrm{l}$ of the culture at that dilution. The same approach was used to find CFU at different dilution used for spotting the culture. In case of $R$. solanacearum, observation of microcolonies was done from $8.0 \mathrm{~h}$ onwards up to $14.0 \mathrm{~h}$ at every $2.0 \mathrm{~h}$ intervals.

\section{CFU from microliter spotting}

After observing the microcolonies under the microscope in their early hours of growth, the petridishes were kept back in the incubator. After overnight growth (in case of $E$. coli) colonies could be visible by naked eyes. In case of $10^{6}$ dilution, average 15 colonies per spot can be observed, while in case of $10^{7}$ dilution average 2.0 colonies per spot were observed. This method proved the observations made earlier through microcolonies counting.

\section{CFU from spread plate method}

In the usual spread plate method to count CFU, $100 \mu \mathrm{l}$ of overnight grown serially diluted bacterial cultures were evenly distributed on the solid growth medium by using a glass spreader. The petridishes were kept in the incubator and after overnight incubation, we observed the colonies by naked eyes. All experiments were performed in triplicates.

\section{Results and Discussion}

We calculated the micro-colonies in an E. coli culture as described in Materials and Methods section. We observed the micro-colonies of $E$. coli by the microscope from ten different spots of different dilutions such as $10^{4}$, $10^{5}, 10^{6}$ from $2.0 \mathrm{~h}$ onwards after incubation till $8.0 \mathrm{~h}$ after every $2 \mathrm{~h}$ interval [SFig1a-1c]. Number of microcolonies observed and CFU calculated are listed in Table 1. After $2.0 \mathrm{~h}$ of incubation, the micro-colonies counts were $2.22 \mathrm{x}$ $10^{9}$ and $3.62 \times 10^{9}$ for the $10^{4}$ and $10^{5}$ dilution spots, respectively. Subsequently, in $4.0 \mathrm{~h}$ of incubation period at $37^{\circ} \mathrm{C}$, the micro-colonies counts were calculated as $1.99 \times 10^{9}, 2.40 \times 10^{9}$ and $4.98 \times 10^{9}$ for the $10^{4}$, $10^{5}$ and $10^{6}$ dilution spots, respectively [Fig. 2a, Table 1]. After $6.0 \mathrm{~h}$ of incubation period, the micro-colonies counts were found out 
$1.8 \times 10^{9}, 2.35 \times 10^{9}$ and $3.04 \times 10^{9}$ for the $10^{4}, 10^{5}$ and $10^{6}$ dilution spots, respectively. While, in $8.0 \mathrm{~h}$ of incubation, the micro-colonies counts were calculated as $1.26 \times 10^{9}, 2.10 \times 10^{9}$ and $3.46 \times 10^{9}$ for the $10^{4}, 10^{5}$ and $10^{6}$ dilution spots, respectively. Further, these micro-colonies counts were confirmed by spot dilution [SFig 2] and plate spreading SFig 3] methods and results showed that almost similar CFU counts were observed with different dilutions at different time periods as listed in Table 3. It supported that microcolonies count can represent the CFU in a culture in case of E. coli.

Likewise, the CFU count using microliter spotting and microcolony counting was done in $R$. solanacearum, a bacterium with generation time more than $\sim 3.0 \mathrm{~h}$, as well. Microcolonies could be observed from $8 \mathrm{~h}$ onwards at $10^{4}$ dilution spotting. However, it could be counted properly after $10 \mathrm{~h}$ of spotting. Fig $2 \mathrm{~b}$ demonstrate microcolonies of $R$. solanacearum after $10 \mathrm{~h}$ of spotting of dilutions such $10^{4}, 10^{5}$, and $10^{6}$. It is pertinent to note that at this hour of growth it is not easy to find colonies in case of $10^{7}$ dilutions as the number of colonies are very low. We observed that the micro-colonies of $R$. solanacearum by the microscope from six different spots of $10^{4}$ dilution and ten different spots of $10^{5}$ and $10^{6}$ each from $8 \mathrm{~h}$ of incubation till $14 \mathrm{~h}$ after every $2 \mathrm{~h}$ interval [SFig4a4c]. Number of microcolonies observed and CFU calculated are listed in Table 2. After $8.0 \mathrm{~h}$ of incubation, the micro-colonies counts were $2.52 \mathrm{x}$ $10^{9}, 3.66 \times 10^{9}$ and $4.84 \times 10^{9}$ for the $10^{4}, 10^{5}$ and $10^{6}$ dilution spots, respectively. Subsequently, in $10.0 \mathrm{~h}$ of incubation period at $28^{\circ} \mathrm{C}$, the micro-colonies counts were calculated as $2.60 \times 10^{9}, 3.46 \times 10^{9}$ and $5.07 \times 10^{9}$ for the $10^{4}, 10^{5}$ and $10^{6}$ dilution spots, respectively [Table 2]. After $12.0 \mathrm{~h}$ of incubation period, the micro-colonies counts were found out $3.92 \times 10^{9}, 4.57 \times 10^{9}$ and $4.61 \times 10^{9}$ for the $10^{4}, 10^{5}$ and $10^{6}$ dilution spots respectively. Similar results were obtained with plate spreading method [Table 3, SFig 5]. We further counted 10 spots for $10^{6}$ and $10^{7}$ dilutions each, as well as 10 plates each of dilutions $10^{6}$ and $10^{7}$ of E. coli and the observation revealed that the co-efficient of variance was comparable by both spotting method and the dilution plating method, respectively [Table 4]. The size of micro-colonies has increased at different time periods, however, these results show that there is a similarity of calculated number of micro-colonies at different time periods [Fig. 3]. It supported that microcolonies count could represent the CFU in a culture in case of $R$. solanacearum. In $R$. solanacearum, the microcolonies grow in size, exhibit twitching motility and aggregate together. Twitching motility is, however, absent in E. coli.

Serial dilution is an easy and convenient approach to measure viable bacterial concentration in a culture. In early days of microbiology, enumerating bacterial number in a culture was made in test tubes by finding out the minimum dilution at which no bacterial growth was observed [11]. Usually, turbidity development in the medium used to be measured as bacterial growth [SFig 6]. Later with the use of agar, colony formation by bacteria could be observed on solid agar medium [12]. With the availability of micropipettes, which can pipette out liquids of volume 1.0 or 2.0 $\mu 1$ with accuracy, it became an aid for us to think of counting bacteria in $5 \mu 1$ volume.

The findings that microcolonies number remain similar at various point of time at a specific dilution supported that the method can be used to count bacteria in a culture by observing micro-colonies. In contrast to the conventional spread plate method, calculating CFU by dilution spotting is both time-saving and economical. While we could calculate $R$. solanacearum and E. coli CFU using dilution spotting at $10 \mathrm{hrs}$ and $4 \mathrm{hrs}$ respectively, we could obtain 
similar results of $R$. solanacearum and E. coli using spread plate method at only $48 \mathrm{hrs}$ and $16 \mathrm{hrs}$ of incubation respectively.

Dilution spotting and subsequent back calculations are a fast and easy technique to enumerate bacterial population in a culture. Besides, CFU can be estimated from different dilutions, such as, $10^{4}, 10^{5}, 10^{6}$ and $10^{7}$. Counting CFU from $10^{4}$ and $10^{5}$ dilution by spread plate method is difficult because the colonies merged with each other due to their high number. Also less variation of CFU numbers across different spots of same dilution is observed. A dilution spot of $10^{5}$ was the most convenient in counting microcolonies. Phase-contrast micrographs not only eliminates the use of stain to distinguish between live and dead cells but also help us identify contaminants to some extent. The advantages to this technique are many, but it too has some cons. Phase-contrast microscope itself comes with a hefty price tag but this method of CFU counting can be performed using any microscope that comes with a scale to extrapolate. Also, this technique demands manual labor; albeit lesser labor-intensive than the traditional counting ways.

\section{Acknowledgements}

SB is thankful for the JRF/SRF fellowship from the UGC-NFSC, GoI, New Delhi. MY is thankful to RA fellowship from the SERB-DST grant (CRG/2020/002651) awarded to AK. SS is thankful to CSIR, GoI, New Delhi for the JRF/SRF fellowship. SJG is thankful for the JRF fellowship from the DBT, GoI New Delhi grant (BT/PR41637/NER/95/1753/2021) awarded to SKR, and also to Tezpur University for the Institutional fellowship. S Begum is thankful to Tezpur University for the institutional fellowship. AJ is thankful to UGC, GoI, New Delhi for the NET-JRF fellowship.

\section{Funding}

The authors would like to acknowledge DBT for the MSc project grants for the consumables and DBT-UExcel/NER for the equipment

\section{Conflict of interest}

We declare that there is no conflict of interest among the authors of this manuscript.

\section{References}

1. Jett BD, Hatter KL, Huycke MM, Gilmore MS (1997) Simplified agar plate method for quantifying viable bacteria. BioTechniques 23(4):648-650. https://doi.org/10.2144/97234BM22

2. McNulty JJ, Dunn JJ (1999) High-throughput transformation and plating using petristrips. BioTechniques 26(3):390-392. https://doi.org/10.2144/99263BM04 
3. Yadav M, Rathore JS (2022) Functional and transcriptional analysis of chromosomal encoded hipBAXn2 type II toxin-antitoxin (TA) module from Xenorhabdus nematophila. Microbial Pathogenesis 162:105309. https://doi.org/10.1016/J.MICPATH.2021.105309

4. Kumar R, Barman A, Jha G, Ray SK (2013) Identification and establishment of genomic identity of Ralstonia solanacearum isolated from a wilted chilli plant at Tezpur, North East India. Curr Sc 105(11):1571-1578.

5. Xu P, Iwata M, Leong S, Sequeira L (1990) Highly virulent strains of Pseudomonas solanacearum that are defective in extracellular-polysaccharide production. J Bacteriol 172(7):3946. https://doi.org/10.1128/JB.172.7.3946-3951.1990

6. Hsieh K, Zec HC, Chen L, Kaushik AM, MacH KE, Liao JC, Wang TH (2018) Simple and Precise Counting of Viable Bacteria by Resazurin-Amplified Picoarray Detection. In Analytical Chem 90(15):9449-9456. https://doi.org/10.1021/acs.analchem.8b02096

7. Koch AL (2014) Growth Measurement. Methods for Gen \& Mol Microbiol 172-199. https://doi.org/10.1128/9781555817497.CH9

8. Pan H, Zhang Y, He GX, Katagori N, Chen H (2014) A comparison of conventional methods for the quantification of bacterial cells after exposure to metal oxide nanoparticles. BMC Microbiol 14(1):222. https://doi.org/10.1186/S12866-014-0222-6

9. Stevenson K, McVey AF, Clark IBN, Swain PS, Pilizota T (2016) General calibration of microbial growth in microplate readers. Sc Reports 6(1):1-7. https://doi.org/10.1038/srep38828

10. Ray SK, Kumar R, Peeters N, Boucher C, Genin S (2015) rpoN1, but not rpoN2, is required for twitching motility, natural competence, growth on nitrate, and virulence of Ralstonia solanacearum. Frontiers in Microbiol 6:229. https://doi.org/10.3389/FMICB.2015.00229/ABSTRACT

11. Koch R. (1883) Über die neuen Untersuchungsmethoden zum Nachweis der Mikrokosmen in Boden, Luft und Wasser. 1.

12. Breed RS, Dotterrer WD (1916) The number of colonies allowable on satisfactory agar plates. J Bacteriol 1(3):321. https://doi.org/10.1128/jb.1.3.321-331.1916 


\section{Figures}

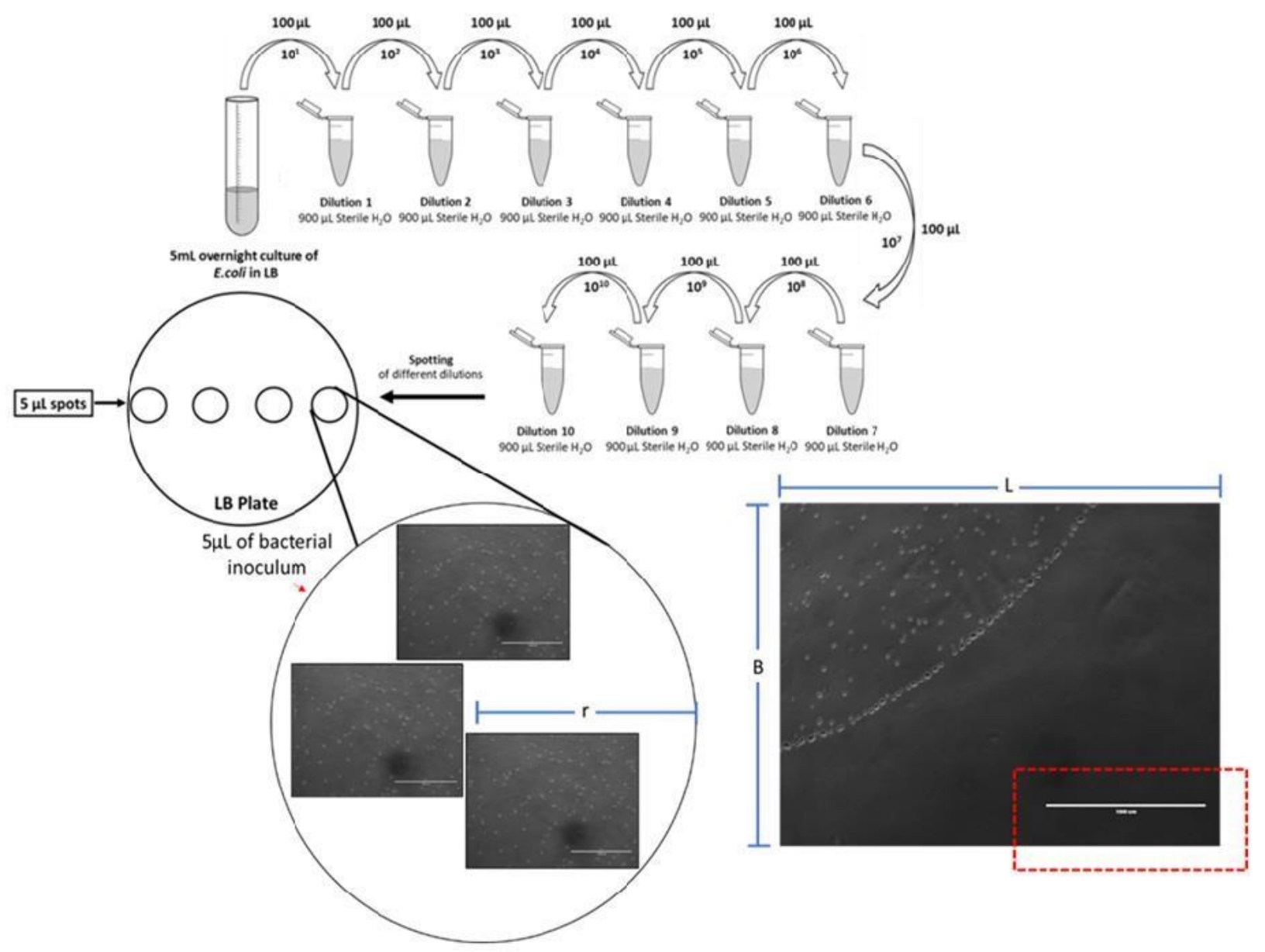

Fig. 1: Schematic representation of the microcolonies counting method

In a given bacterial culture considered for counting CFU, dilution of the culture was performed as shown in the figure. $100 \mu \mathrm{l}$ of the culture was added to $900 \mu \mathrm{l}$ of water to make it 10 -fold $\left(10^{1}\right)$ dilution, from the diluted culture $100 \mu \mathrm{l}$ was taken and added to $900 \mu \mathrm{l}$ of water to dilute it another 10 times. The culture was diluted $10^{10}$ fold. Only $5 \mu \mathrm{l}$ from different dilutions were spotted on a LB-Agar plate in case of E. coli. Approximately, ten such spots can be made on a Petri-plates (90 mm diameter). After $2.0 \mathrm{~h}$ of incubation, micro-colonies were observed at 40x magnification. Three different sections of $10^{4}$ dilution spotted have been shown here. Distinct colonies could be observed. 

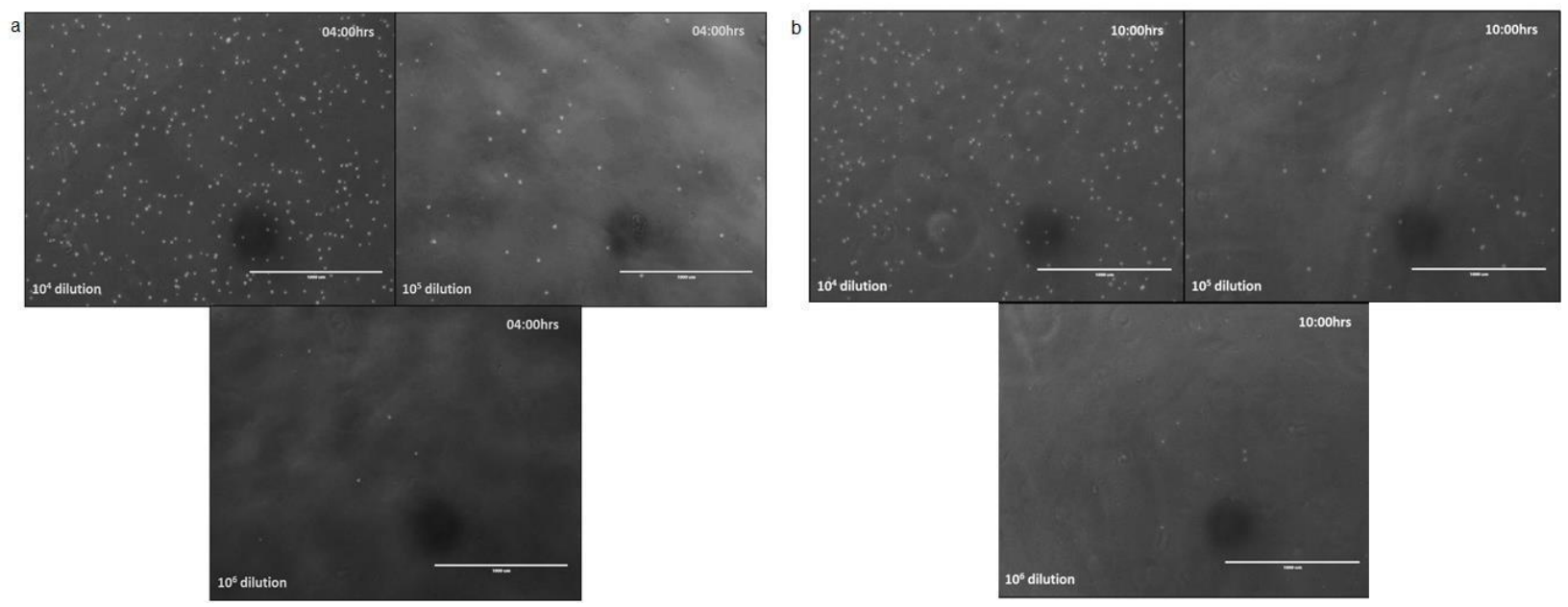

Fig 2a. E. coli microcolonies number observed at different time points

At $2.0 \mathrm{~h}, 4.0 \mathrm{~h}, 6.0 \mathrm{~h}$ and $8.0 \mathrm{~h}$ duration microcolonies were observed for different dilutions such as $10^{4}, 10^{5}$ and $10^{6}$ of E. coli culture. Counted microcolonies found to be similar at different time points though there is growth in colony size. Further, number of CFU estimated by counting the microcolonies at different dilutions is observed to be similar.

Fig 2b. R. solanacearum microcolonies number observed at different time points

At $8.0 \mathrm{~h}, 10.0 \mathrm{~h}, 12.0 \mathrm{~h}$ and $14.0 \mathrm{~h}$ duration microcolonies were observed for different dilutions such as $10^{4}$, $10^{5}$ and $10^{6}$ of R. solanacearum culture. Counted microcolonies found to be similar at different time points though there is growth in colony size. Further, number of CFU estimated by counting the microcolonies at different dilutions is observed to be similar. 
bioRxiv preprint doi: https://doi.org/10.1101/2022.01.26.477842; this version posted January 28, 2022. The copyright holder for this preprint (which was not certified by peer review) is the author/funder. All rights reserved. No reuse allowed without permission.

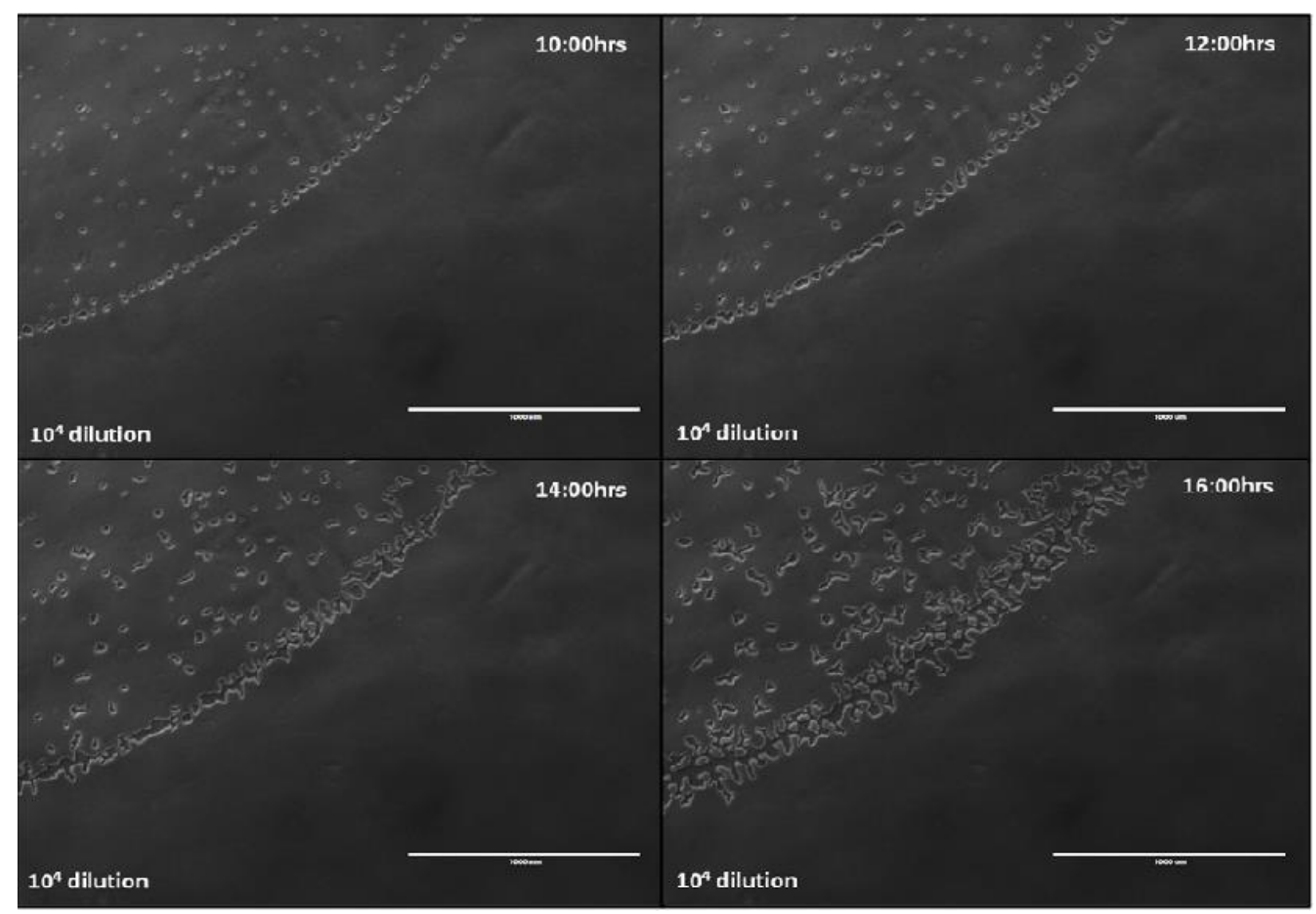

Fig. 3. R. solanacearum microcolonies at different dilutions

$R$. solanacearum microcolonies observed at different dilutions such as $10^{4}, 10^{5}, 10^{6}$ and $10^{7}$ after at $10 \mathrm{~h}$ incubation. Microcolony formed are distinctly observed at different dilutions. The number are decreasing with increase in dilution. This suggest that CFU count is possible after $10 \mathrm{~h}$ of incubation in case of $R$. solanacearum. 
Table 1: CFU calculated in $E$. coli culture by micro-colony counting method

\begin{tabular}{ccl}
\hline Dilution & Time (h) & CFU/mL \\
\hline \multirow{2}{*}{$10^{4}$} & 2 & $2.22 \times 10^{9}$ \\
& 4 & $1.99 \times 10^{9}$ \\
& 6 & $1.80 \times 10^{9}$ \\
& 8 & $1.26 \times 10^{9}$ \\
\hline \multirow{2}{*}{$10^{5}$} & 2 & $3.62 \times 10^{9}$ \\
& 4 & $2.40 \times 10^{9}$ \\
& 6 & $2.35 \times 10^{9}$ \\
\multirow{2}{*}{$10^{6}$} & 8 & $2.10 \times 10^{9}$ \\
& 4 & $4.98 \times 10^{9}$ \\
& 6 & $3.04 \times 10^{9}$ \\
& 8 & $3.46 \times 10^{9}$ \\
\hline
\end{tabular}

Table 2: CFU calculate in $R$. solanacearum by micro-colony counting method

\begin{tabular}{ccc}
\hline Dilution & Time (h) & CFU/ml \\
\hline \multirow{2}{*}{$10^{4}$} & 8 & $2.52 \times 10^{9}$ \\
& 10 & $2.60 \times 10^{9}$ \\
& 12 & $3.92 \times 10^{9}$ \\
& 14 & $1.26 \times 10^{9}$ \\
\hline \multirow{2}{*}{$10^{5}$} & 8 & $3.66 \times 10^{9}$ \\
& 10 & $3.46 \times 10^{9}$ \\
& 12 & $4.57 \times 10^{9}$ \\
$10^{6}$ & 14 & $3.83 \times 10^{9}$ \\
\hline & 8 & $4.84 \times 10^{9}$ \\
& 10 & $5.07 \times 10^{9}$ \\
& 12 & $4.61 \times 10^{9}$ \\
\hline
\end{tabular}


Table 3: Comparison of CFU calculation by $5 \mu$ spotting and $100 \mu \mathrm{l}$ spreading method

\begin{tabular}{|c|c|c|c|c|}
\hline \multirow{2}{*}{ Dilutions } & \multicolumn{2}{|c|}{ R. solanacearum } & \multicolumn{2}{c|}{ E. coli } \\
\cline { 2 - 5 } & Dilution spotting & Spread plate & Dilution spotting & Spread plate \\
\hline $10^{4}$ & $2.1 \times 10^{9}$ cells & & Colonies merge with each other \\
\hline $10^{5}$ & $2.3 \times 10^{9}$ cells & & $6.04 \times 10^{8}$ & $4.5 \times 10^{8}$ \\
\hline $10^{6}$ & $3.5 \times 10^{9}$ & $10^{9}$ & $6.2 \times 10^{8}$ & $7.1 \times 10^{8}$ \\
\hline $10^{7}$ & $7 \times 10^{9}$ & $1.1 \times 10^{9}$ & $1.6 \times 10^{9}$ & $7 \times 10^{8}$ \\
\hline $10^{8}$ & No colonies found & $6.7 \times 10^{8}$ & $4 \times 10^{9}$ & $6.7 \times 10^{8}$ \\
\hline
\end{tabular}


Table 4: Comparison of visible colonies observed by both the methods in $E$. coli

\begin{tabular}{|c|c|c|}
\hline & $10^{6}$ dilution & $10^{7}$ dilution \\
S & $5 \mu \mathrm{L}$ & $\mathbf{5 L \mathrm { L }}$ \\
$\mathbf{P}$ & 20 & 2 \\
& 21 & 1 \\
$\mathbf{O}$ & 11 & 0 \\
$\mathbf{T}$ & 15 & 1 \\
$\mathbf{T}$ & 19 & 1 \\
$\mathbf{I}$ & 16 & 0 \\
N & 9 & 5 \\
G & 14 & 1 \\
& 11 & 3 \\
& 17 & 1 \\
\hline MEAN & 15.3 & 1.5 \\
SD & 4.083844 & 1.509231 \\
CV & 0.266918 & 1.006154 \\
\hline
\end{tabular}

\begin{tabular}{|c|c|c|}
\hline & $10^{6}$ dilution & $10^{7}$ dilution \\
S & $100 \mu \mathrm{L}$ & $100 \mu \mathrm{L}$ \\
P & 121 & 13 \\
& 233 & 13 \\
R & 106 & 30 \\
E & 102 & 13 \\
A & 156 & 14 \\
D & 80 & 19 \\
I & 199 & 21 \\
N & 242 & 29 \\
G & 210 & 23 \\
& 205 & 27 \\
\hline MEAN & 165.4 & 20.2 \\
SD & 59.65121 & 6.860515 \\
CV & 0.360648 & 0.339629 \\
\hline
\end{tabular}

